\title{
Risk Scores and Machine Learning to Identify Patients With Acute Periprosthetic Joints Infections That Will Likely Fail Classical Irrigation and Debridement
}

\begin{abstract}
Marjan Wouthuyzen-Bakker ${ }^{1 *}$, Noam Shohat ${ }^{2,3}$, Javad Parvizi ${ }^{4}$ and Alex Soriano ${ }^{5}$
${ }^{1}$ Department of Medical Microbiology and Infection Prevention, University Medical Center Groningen, University of Groningen, Groningen, Netherlands, ${ }^{2}$ Department of Orthopaedic Surgery, Yitzhak Shamir Medical Center, Zriffin, Israel, ${ }^{3}$ Department of Orthopaedic Surgery, Tel Aviv University, Tel Aviv, Israel, ${ }^{4}$ Department of Orthopaedic Surgery, Rothman Institute at Thomas Jefferson University Hospital, Philadelphia, PA, United States, ${ }^{5}$ Service of Infectious Diseases, Hospital Clínic, University of Barcelona, Barcelona, Spain
\end{abstract}

OPEN ACCESS

Edited by:

Marc Jean Struelens, Université libre de Bruxelles, Belgium

Reviewed by:

Akos Zahar,

HELIOS Klinikum Emil von

Behring, Germany

Jesse Kuiper,

Spaarne Gasthuis, Netherlands

${ }^{*}$ Correspondence:

Marjan Wouthuyzen-Bakker m.wouthuyzen-bakker@umcg.n

Specialty section:

This article was submitted to Infectious Diseases - Surveillance,

Prevention and Treatment, a section of the journal Frontiers in Medicine

Received: 08 April 2020 Accepted: 23 March 2021

Published: 03 May 2021

Citation:

Wouthuyzen-Bakker M, Shohat N, Parvizi J and Soriano A (2021) Risk Scores and Machine Learning to Identify Patients With Acute Periprosthetic Joints Infections That Will Likely Fail Classical Irrigation and Debridement.

Front. Med. 8:550095.

doi: 10.3389/fmed.2021.550095
The most preferred treatment for acute periprosthetic joint infection (PJI) is surgical debridement, antibiotics and retention of the implant (DAIR). The reported success of DAIR varies greatly and depends on a complex interplay of several host-related factors, duration of symptoms, the microorganism(s) causing the infection, its susceptibility to antibiotics and many others. Thus, there is a great clinical need to predict failure of the "classical" DAIR procedure so that this surgical option is offered to those most likely to succeed, but also to identify those patients who may benefit from more intensified antibiotic treatment regimens or new and innovative treatment strategies. In this review article, the current recommendations for DAIR will be discussed, a summary of independent risk factors for DAIR failure will be provided and the advantages and limitations of the clinical use of preoperative risk scores in early acute (post-surgical) and late acute (hematogenous) PJls will be presented. In addition, the potential of implementing machine learning (artificial intelligence) in identifying patients who are at highest risk for failure of DAIR will be addressed. The ultimate goal is to maximally tailor and individualize treatment strategies and to avoid treatment generalization.

Keywords: debridement, implant retention, risk score, machine learning, failure, periprosthetic joint infection

\section{INTRODUCTION}

Success rates of the "classical" debridement, antibiotics, irrigation and implant retention (DAIR) for acute periprosthetic joint infections (PJI) vary widely, ranging from 30 to $90 \%$ (1-5). Apart from a thorough surgical debridement with exchange of modular components, many factors contribute to the success of DAIR; that includes shorter duration of symptoms, lack of patient comorbidities, a low bacterial inoculum and/or degree of inflammation at clinical presentation, a causative microorganism that is susceptible to antibiotics with anti-biofilm properties and many others (6-25). For this reason, being able to identify a category of patients who are likely to fail DAIR is essential, either to choose a different surgical procedure, to intensify antimicrobial 
treatment or to apply new innovative treatment strategies to increase the chance of treatment success. In this overview we will outline the current recommendations for DAIR treatment and discuss the limitations of these recommendations. In addition, we will address preoperative risk classification systems and the potential of machine learning to predict DAIR failure. These latter two show great potential to be used in clinical practice and may aid in clinical decision making.

\section{WHO SHOULD RECEIVE DAIR ACCORDING TO THE IDSA GUIDELINES}

Since many different factors have been identified in literature as independent predictors for DAIR failure (Table 1) (6-25), it is a great challenge to select those patients who are the best candidates for DAIR. According to the IDSA guidelines published in 2013 (26), a DAIR is advised for patients with acute PJI, defined as a symptom duration of $<3$ weeks or, in case of early post-surgical infections, within 4 weeks of index arthroplasty. In addition, the prosthesis needs to be well-fixed, a sinus tract should be absent and the microorganism needs to be susceptible to oral antimicrobial agents with anti-biofilm activity. If these conditions are met, a DAIR is recommended, and in other situations revision of the implant is advised. Although this approach seems legitimate, it entails important limitations. First, it excludes a large subgroup of patients that may still benefit from DAIR. For example, in post-surgical cases it is advised to remove the infected implant when the index arthroplasty occurred more than 4 weeks ago. However, the process of mature biofilm formation varies substantially according to the type of causative microorganism and the inoculum size that contaminates the joint during surgery $(27,28)$. To therefore, exclude these patients as a candidate for a DAIR procedure is not justified. Indeed, Löwik et al. demonstrated an acceptable outcome of DAIR in patients presenting more than 4 weeks after the index arthroplasty as long as DAIR was performed within 4 week after the onset of symptoms and modular components were exchanged (29). In this category of patients, the prosthesis could still be retained in around $80 \%$ of patients without the need for life long suppressive antibiotic treatment. A second limitation of the IDSA recommendation concerning the indication for DAIR is the lack of distinction between early acute (post-surgical) and late acute (hematogenous) PJIs. This distinction may be critical, since several studies demonstrated a worse outcome in late acute PJIs treated with DAIR compared to early acute PJIs, in particular when caused by staphylococci $(8,13,23,30)$. Considering the difference in pathogenesis, and the chance of continuous seeding to the prosthetic joint in case of hematogenous infections (e.g., endocarditis), it is reasonable to assume that these infections should be approached differently as well. A third limitation of the IDSA guideline, is that the causative microorganism(s) and its susceptibility to antibiotics are often not known prior to surgery. A final limitation is the fact that implant- and host-related factors are not included in the decision-making model to determine appropriateness of DAIR. This may result in misclassifying a patient as a good candidate for DAIR while existing comorbidities may expose the patient to an increased risk for complications and failure. In addition, as the microorganism and its susceptibility to antibiotics is often not known prior to surgery, these implant- and host-related factors are of utmost importance to take into consideration.

\section{PREOPERATIVE RISK SCORES TO PREDICT DAIR FAILURE}

To identify patients who are likely to fail DAIR, two preoperative risk scores have been proposed in literature; one for early acute (post-surgical) and one for late acute (hematogenous) PJIs (8, 16). These risk scores include only those variables that are known preoperatively without taking into account the causative microorganism and its susceptibility to antibiotics, mimicking the situation mostly encountered in clinical practice.

\section{KLIC-score for Early Acute (Post-Surgical) PJI}

In 2015, Tornero et al. published the KLIC-score as preoperative risk score for predicting DAIR failure in early acute PJI (16). The authors of this study examined a cohort of 222 patients who were within 3 months after the index surgery and who had no more than 3 weeks of symptoms prior to DAIR. DAIR failure was defined as the need for a second DAIR, implant removal, suppressive antibiotic treatment or infection-related death within 60 days after the initial irrigation and debridement. They analyzed in a univariate model several variables that were known preoperatively, like host-related factors, duration of symptoms, characteristics of the infected implant and serum inflammatory parameters, and developed a risk stratification score according to the beta-coefficients of the multivariate analysis (Figure 1A). Chronic Kidney disease, Liver cirrhosis, the Index surgery (revision surgery or prosthesis indicated for a fracture), a Cemented prosthesis and a C-reactive protein $>115$ $\mathrm{mg} / \mathrm{l}$ (KLIC) appeared to be the most prominent preoperative variables associated with failure. The score demonstrated $100 \%$ DAIR failure when having a preoperative score of more than six, and $4.5 \%$ when having a score lower than two. After this publication, three additional studies from other institutions validated the KLIC-score in their cohort of patients $(18,31,32)$. All three institutions demonstrated the predictive power of the KLIC-score in patients with a very low or a very high score, but the score was less useful in patients with average scores. In addition, one study identified that other variables appeared to be more predictive in their cohort of patients compared to those defined in the KLIC (18), stressing the importance of differences in local epidemiology when implementing risk scores from an external cohort of patients.

\section{CRIME80-score for Late Acute (Hematogenous) PJI}

Following the KLIC-score, Wouthuyzen-Bakker et al. performed the same statistical analysis in a large multicenter cohort of 340 patients with late acute PJIs (8). Late acute PJI was defined as the appearance of acute symptoms of infection occurring 
TABLE 1 | Summary of studies depicting independent predictors of DAIR failure in acute PJls by using multivariate analysis.

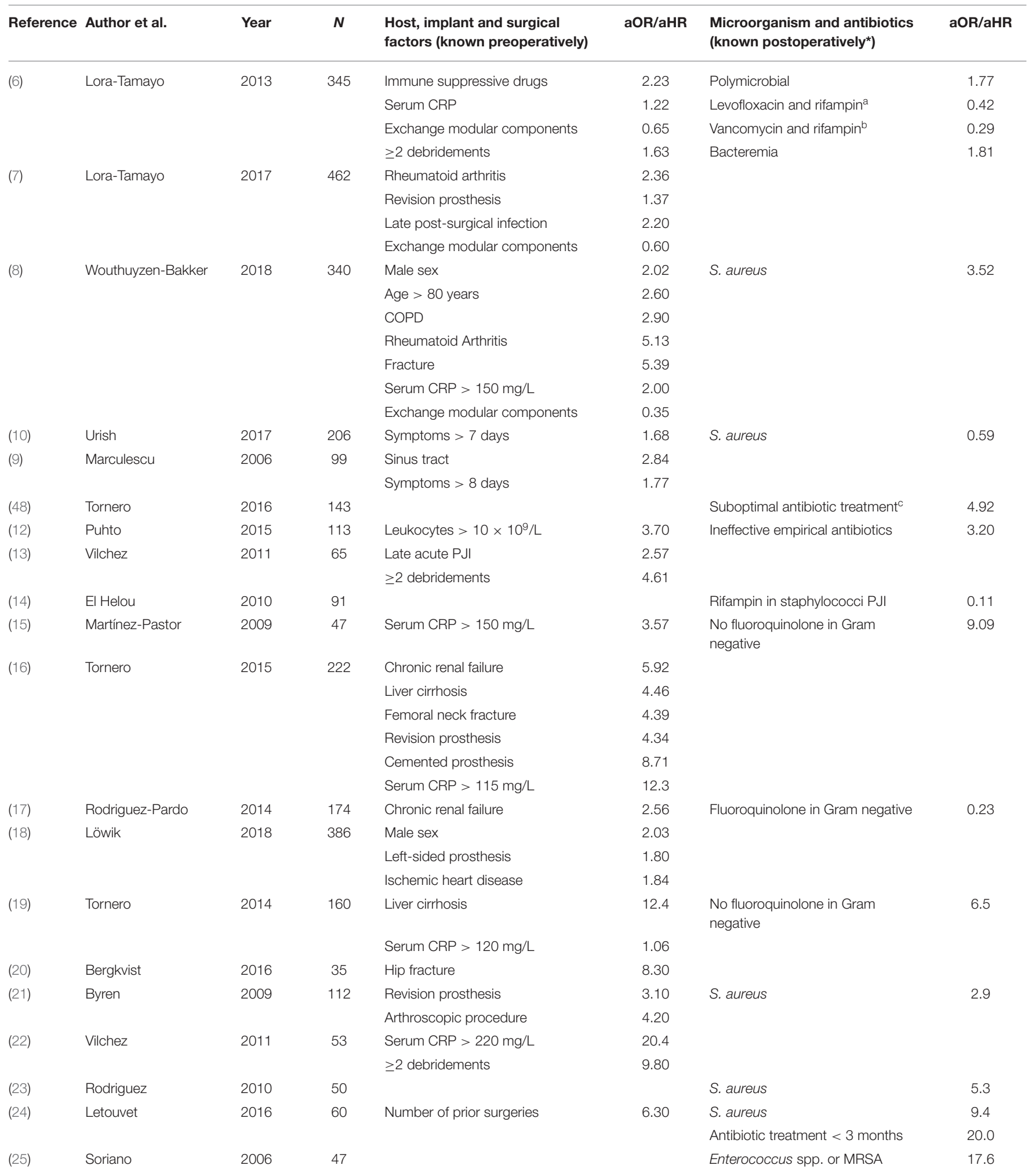

*The presence of bacteremia, the causative microorganism and its susceptibility to antibiotics are sometimes known prior to DAIR, but in most cases not.

a Sub-group analysis of patients with a post-surgical PJI due to methicillin-susceptible S. aureus (MSSA).

bSub-group analysis of patients with a post-surgical PJl due to methicillin-resistant S. aureus (MRSA).

${ }^{c}$ No rifampin for Gram positives and no fluoroquinolone for Gram negatives.

CRP, C-reactive protein; COPD, Chronic Obstructive Pulmonary Disease. 


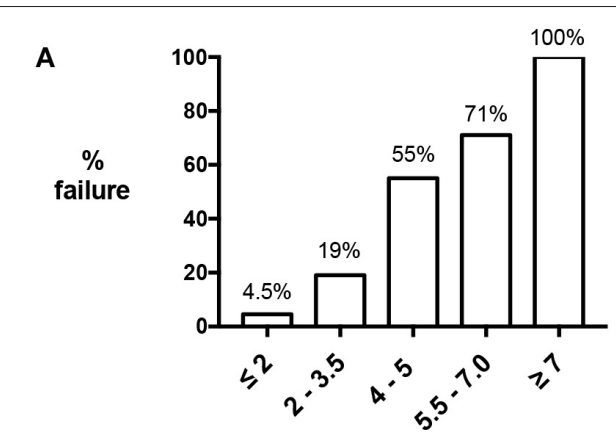

B

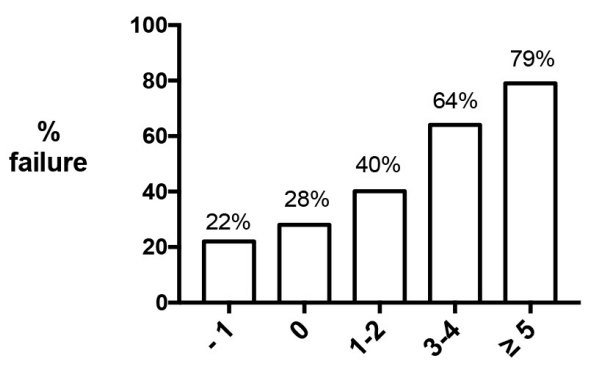

K Chronic renal failure (Kidney)

L Liver cirrhosis

I Index surgery: indication prosthesis: fracture OR 1.5 revision prothesis

C Cemented prosthesis CRP $>115 \mathrm{mg} / \mathrm{L}$
2

1.5

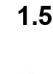

2
C COPD

CRP > $150 \mathrm{mg} / \mathrm{L}$

R Rheumatoid arthritis

I Indication prosthesis: fracture

M Male

E Exchange of mobile components

80 Age $>80$ years
2
1
3
3
1
-1
2

FIGURE 1 | Preoperative risk scores for DAIR failure. KLIC-score for predicting DAIR failure in early acute (postsurgical) PJI (A) and CRIME80-score for predicting DAIR failure in late acute (hematogenous) PJI (B).

more than 3 months after the index arthroplasty, in a prior asymptomatic prosthetic joint. Patients with a sinus tract and/or patients with symptoms existing for longer than 3 weeks before DAIR were excluded. In contrast to the study of Tornero et al., a second DAIR procedure was not considered as failure, and failure could occur even 60-days after the initial debridement. In addition, the authors also included the exchange of mobile components as a valid preoperative variable, as the possibility to exchange it can be known prior to surgery as well. According to this analysis, Chronic obstructive pulmonary disease, a Creactive protein $>150 \mathrm{mg} / \mathrm{L}$, Rheumatoid arthritis, fracture as Indication for the prosthesis, Male sex, not Exchanging the mobile components and an age $>\mathbf{8 0}$ years (CRIME80), were the strongest preoperative variables associated with failure (Figure 1B). The strength of prediction of the CRIME80-score was lower than the KLIC-score, starting with a baseline failure rate of $22 \%$, and increasing to $79 \%$ with a score higher than four. It is important to note that the isolation of Staphylococcus aureus was one of the major predictors of failure in the late acute cohort. When $S$. aureus was the causative microorganism, the baseline failure rate was $43 \%$, and the preoperative variables turned out to be less predictive in these cases. For this reason, the authors stress the importance of isolating the microorganism prior to deciding the surgical procedure. Unlike the KLIC-score, the CRIME80-score has not yet been validated in an external cohort of patients.

\section{Potential of Machine Learning (Artificial Intelligence) in Predicting DAIR Failure}

Considering the complex interplay of factors associated with DAIR failure, regular statistical methods lack the finesse for more accurate and individualized predictions. The advantage of machine learning over regular statistical methods, like multivariate analysis, is its ability to actually learn from data input. Where multivariate analysis examines the correlation of variables and the strength of these correlations, machine learning learns from observations by using decision trees. The subsequently created algorithm is then able to process new input that has not been seen before. By this means, machine learning models are able to process more complex data, and by building precision models they are able to make more accurate predictions. Machine learning has become more and more popular in infection management (33). Recently, Shohat et al. used random forest analysis as a machine learning model to predict DAIR failure (34). The authors of this study analyzed more than 1,000 patients that underwent irrigation and debridement of a hip or knee prosthesis for acute PJI. The created algorithm had good discriminatory power, with an area under the curve of 0.74 . Cross-validation, a model validation technique assessing the ability to process an independent dataset, showed similar probabilities, indicating a high accuracy of the model. Although the model still needs to be validated in an external cohort of patients, the created algorithm has great potential 
to be used in daily practice by easily entering patient data in a computer-based software or smartphone application, and may aid in clinical decision making and patient counseling. As the causative microorganism is of great influence on treatment outcome $(7,8)$, the authors of this study decided to include this variable in the analysis as well. Although its inclusion improves its predictive power, the microorganism needs to be entered to ensure the highest accuracy of the model, and thus, ideally should be known prior to surgery. The same holds for the presence or absence of bacteremia.

\section{TO TAILOR AND INDIVIDUALIZE TREATMENT STRATEGIES}

The described preoperative risk scores and machine learning model, can be applied in daily clinical practice, and may aid in the decision making process. When a patient has a high a priori risk for DAIR failure, immediate implant removal should be considered; not only to avoid surgery that is very likely to fail, but also to reduce the adverse effect of DAIR on subsequent surgical procedures $(35,36)$. Wouthuyzen-Bakker et al. analyzed the treatment outcome of immediate implant removal vs. DAIR in late acute PJIs by matching patients according to their preoperative CRIME80 score (37). The authors found that implant removal resulted in $83 \%$ treatment success in patients with a CRIME80 score $\geq 3$, while the success was only $35 \%$ when treated with DAIR. No clear difference was observed between one- and two-stage exchange arthroplasties. Although a high CRIME80 score was logically associated with the presence of more comorbidities and old age, immediate implant removal was associated with a lower-instead of highermortality rate compared to DAIR. These data suggest that immediate implant removal is safe, even though the surgery in general is more aggressive.

A promising technique to potentially increase the success rate of DAIR, especially for difficult to treat microorganisms (e.g., multidrug resistant Gram negatives or rifampin resistant staphylococci), is to locally inject a selected cocktail of bacteriophages during surgery. Although future studies are needed to endorse this practice, its clinical success has been described as salvage therapy in relapsing S. aureus PJI (38). A main disadvantage though, is that the microorganism(s) causing the infection not only needs to be known prior to surgery, but the corresponding targeted bacteriophages need to be produced in the laboratory, before they can be applied. Since delaying DAIR increases the risk of treatment failure, the use of bacteriophage therapy for this indication is therefore, probably less feasible.

\section{REFERENCES}

1. Faschingbauer M, Kappe T, Trubrich A, Bieger R, Reichel H. Retention of the prosthesis in early periprosthetic infection after total hip arthroplasty. Z Orthop Unfall. (2015) 153:192-7. doi: 10.1055/s-0035-15 45803
When a DAIR has been performed in a patient with a low preoperative risk score for failure, but the infection turns out to be caused by a microorganism that is resistant to biofilm active drugs, antibiotic duotherapy can be considered, particularly during the initial period. Adding fosfomycin to the antibiotic regimen as a second drug in infections with multidrug resistant Gram negatives or Gram positive microorganisms, or adding daptomycin for Gram positive infections, have shown great promise: both of the latter antibiotics have shown good antibiofilm properties in vitro and in vivo when used as part of a combination treatment (39-45). An alternative option is life-long antibiotic suppressive therapy, especially if patients are not eligible for additional surgery. According to a recent large multicenter cohort study with a follow-up period of 5 years, PJI can be controlled with antibiotic suppressive therapy in around $50 \%$ of cases (46). Another alternative strategy would be to apply new and more innovative treatments to control infection, like applying subcutaneous antibiotics for patients who do not tolerate oral antibiotics or for infections caused by multidrug resistant bacteria that lack an oral alternative. This proof of concept was demonstrated by Ferry et al. and was successful in 6 out of 10 patients (47). Considering the low chance of success for both treatment strategies, isolating the microorganism prior to surgery and choosing for implant removal in high risk patients would be preferable.

\section{CONCLUSION}

Selecting those patients who are good candidates for a DAIR procedure is essential. Current IDSA recommendations for DAIR entail important limitations, and tools that also take into account other variables that are associated with DAIR failure are needed. Preoperative risk scores like the KLIC-score for early acute (postsurgical) and CRIME80-score for late acute (hematogenous) PJI could be helpful, especially when the microorganism is not known prior to surgery. In addition, machine learning shows great potential to predict failure more accurately compared to regular statistical methods. Implementing the aforementioned tools in daily care will help physicians tailor and individualize treatment strategies. Both described risk classification systems as well as the recently published machine learning model need clinical evaluation in larger external cohorts of patients to validate its predictive power.

\section{AUTHOR CONTRIBUTIONS}

All authors listed have made a substantial, direct and intellectual contribution to the work, and approved it for publication.

2. Duque A, Post Z, Lutz R, Orozco F, Pulido S, Ong A. Is there still a role for irrigation and debridement with liner exchange in acute periprosthetic total knee infection? J Arthroplasty. (2017) 32:12804. doi: 10.1016/j.arth.2016.10.029

3. Fehring TK, Odum SM, Berend KR, Jiranek WA, Parvizi J, Bozic KJ, et al. Failure of irrigation and débridement for early 
postoperative periprosthetic infection. Clin Orthop Relat Res. (2013) 471:250-7. doi: 10.1007/s11999-012-2373-9

4. Westberg M, Grøgaard B, Snorrason F. Early prosthetic joint infections treated with debridement and implant retention. Acta Orthop. (2012) 83:22732. doi: 10.3109/17453674.2012.678801

5. Tsang S, Ting J, Simpson A, Gaston P. Outcomes following debridement, antibiotics and implant retention in the management of periprosthetic infections of the hip: a review of cohort studies. Bone Joint J. (2017) 99B:1458-66. doi: 10.1302/0301-620X.99B11.BJJ-2017-0088.R1

6. Lora-Tamayo J, Murillo O, Iribarren JA, Soriano A, Sánchez-Somolinos M, Baraia-Etxaburu JM, et al. A large multicenter study of methicillinsusceptible and methicillin-resistant Staphylococcus aureus prosthetic joint infections managed with implant retention. Clin Infect Dis. (2013) 56:18294. doi: $10.1093 / \mathrm{cid} / \mathrm{cis} 746$

7. Lora-Tamayo J, Senneville É, Ribera A, Bernard L, Dupon M, Zeller V, et al. The not-so-good prognosis of streptococcal periprosthetic joint infection managed by implant retention: the results of a large multicenter study. Clin Infect Dis. (2017) 64:1742-52. doi: 10.1093/cid/cix227

8. Wouthuyzen-Bakker M, Sebillotte M, Lomas J, Taylor A, Palomares EB, Murillo O, et al. Clinical outcome and risk factors for failure in late acute prosthetic joint infections treated with debridement and implant retention. J Infect. (2019) 78:40-7. doi: 10.1016/j.jinf.2018.07.014

9. Marculescu CE, Berbari EF, Hanssen AD, Steckelberg JM, Harmsen SW, Mandrekar JN, et al. Outcome of prosthetic joint infections treated with debridement and retention of components. Clin Infect Dis. (2006) 142:4718. doi: 10.1086/499234

10. Urish KL, Bullock AG, Kreger AM, Shah NB, Jeong K, Rothenberger SD, et al. A multicenter study of irrigation and debridement in total knee arthroplasty periprosthetic joint infection: treatment failure is high. J Arthroplasty. (2017) 17:31034-3. doi: 10.1016/j.arth.2017.11.029

11. Koh IJ, Han SB, In Y, Oh KJ, Lee DH, Kim TK, et al. Open debridement and prosthesis retention is a viable treatment option for acute periprosthetic joint infection after total knee arthroplasty. Arch Orthop Trauma Surg. (2015) 135:847-55. doi: 10.1007/s00402-015-2237-3

12. Puhto AP, Puhto T, Niinimäki T, Ohtonen P, Leppilahti J, Syrjälä H. Predictors of treatment outcome in prosthetic joint infections treated with prosthesis retention. Int Orthop. (2015) 39:1785-91. doi: 10.1007/s00264-015-2819-2

13. Vilchez F, Martínez-Pastor JC, García-Ramiro S, Bori G, Tornero E, García E, et al. Efficacy of debridement in hematogenous and early postsurgical prosthetic joint infections. Int J Artif Organs. (2011) 34:8639. doi: 10.5301/ijao.5000029

14. El Helou OC, Berbari EF, Lahr BD, Eckel-Passow JE, Razonable RR, Sia IG, et al. Efficacy and safety of rifampin containing regimen for staphylococcal prosthetic joint infections treated with debridement and retention. Eur J Clin Microbiol Infect Dis. (2010) 29:961-7. doi: 10.1007/s10096-010-0952-9

15. Martínez-Pastor JC, Munoz-Mahamud E, Vilchez F, García-Ramiro S, Bori $\mathrm{G}$, Sierra J, et al. Outcome of acute prosthetic joint infections due to gramnegative bacilli treated with open debridement and retention of the prosthesis. Antimicrob Agents Chemother. (2009) 53:4772-7. doi: 10.1128/AAC.00188-09

16. Tornero E, Morata L, Martinez-Pastor JC, Bori G, Climent C, Garcia-Velez $\mathrm{DM}$, et al. KLIC-score for predicting early failure in prosthetic joint infection treated with debridement, implant retention and antibiotics. Clin Microbiol Infect. (2015) 21:786. doi: 10.1016/j.cmi.2015.04.012

17. Rodríguez-Pardo D, Pigrau C, Lora-Tamayo J, Soriano A, Del Toro MD, Cobo J, et al. Gram-negative prosthetic joint infection: outcome of a debridement, antibiotics and implant retention approach. A large multicentre study. Clin Microbiol Infect. (2014) 20:O911-9. doi: 10.1111/1469-0691.12649

18. Löwik CAM, Jutte PC, Tornero E, Ploegmakers JJW, Knobben BAS, de Vries AJ, et al. Predicting failure in early acute prosthetic joint infection treated with debridement, antibiotics and implant retention: external validation of the KLIC-score. J Arthroplasty. (2018) 33:258287. doi: 10.1016/j.arth.2018.03.041

19. Tornero E, Martínez-Pastor JC, Bori G, García-Ramiro S, Morata L, Bosch J, et al. Risk factors for failure in early prosthetic joint infection treated with debridement. Influence of etiology and antibiotic treatment. J Appl Biomater Funct Mater. (2014) 12:129-34. doi: 10.5301/jabfm.5000209

20. Bergkvist M, Mukka SS, Johansson L, Ahl TE, Sayed-Noor AS, Sköldenberg OG, et al. Debridement, antibiotics and implant retention in early periprosthetic joint infection. Hip Int. (2016) 26:138-43. doi: 10.5301/hipint.5000328

21. Byren I, Bejon P, Atkins BL, Angus B, Masters S, McLardy-Smith P, et al. One hundred and twelve infected arthoplasties treated with 'DAIR' (debridement, antibiotics and implant retention): antibiotic duration and outcome. $J$ Antimicrob Chemother. (2009) 63:1264-71. doi: 10.1093/jac/dkp107

22. Vilchez F, Martínez-Pastor JC, Garcia-Ramiro S, Bori G, Maculé F, Sierra J, et al. Outcome and predictors of treatment failure in early post-surgical prosthetic joint infections due to Staphylococcus aureus treated with debridement. Clin Microbiol Infect. (2011) 17:439-44. doi: 10.1111/j.1469-0691.2010.03244.x

23. Rodríguez D, Pigrau C, Euba G, Cobo J, García-Lechuz J, Palomino J, et al. Acute haematogenous prosthetic joint infection: prospective evaluation of medical and surgical management. Clin Microbiol Infect. (2010) 16:178995. doi: 10.1111/j.1469-0691.2010.03157.x

24. Letouvet B, Arvieux C, Leroy H, Polard JL, Chapplain JM, Common H, et al. Predictors of failure for prosthetic joint infections treated with debridement. Med Mal Infect. (2016) 46:39-43. doi: 10.1016/j.medmal.2015.11.007

25. Soriano A, Garcia S, Bori G, Almela M, Gallart X, Macule F, et al. Treatment of acute post-surgical infection of joint arthroplasty. Clin Microbiol Infect. (2006) 12:930-3. doi: 10.1111/j.1469-0691.2006.01463.x

26. Osmon DR, Berbari EF, Berendt AR, Lew D, Zimmerli W, Steckelberg JM, et al. Diagnosis and management of prosthetic joint infection: clinical practice guidelines by the Infectious Diseases Society of America. Clin Infect Dis. (2013) 56:1-25. doi: 10.1093/cid/cis966

27. Lovati A, Bottagisio M, de Vecchi E, Gallazzi E, Drago L. Animal models of implant-related lowgrade infections. A twenty year review. Adv Exp Med Biol. (2017) 971:29-50. doi: 10.1007/5584_2016_157

28. Vidlak D, Kielian T. Infectious dose dictates the host response during Staphylococcus aureus orthopedic-implant biofilm infection. Infect Immun. (2016) 84:1957-65. doi: 10.1128/IAI.00117-16

29. Löwik CAM, Parvizi J, Jutte PC, Zijlstra WP, Knobben BAS, Xu C, et al. Debridement, antibiotics and implant retention is a viable treatment option for early periprosthetic joint infection presenting more than four weeks after index arthroplasty. Clin Infect Dis. (2019) 71:630-6. doi: 10.1093/cid/ciz867

30. Shohat N, Goswami K, Tan TL, Fillingham Y, Parvizi J. Increased failure after irrigation and debridement for acute hematogenous periprosthetic joint infection. J Bone Joint Surg Am. (2019) 101:696-703. doi: 10.2106/JBJS.18.00381

31. Duffy S, Ahearn N, Darley ES, Porteous AJ, Murray JR, Howells NR. Analysis of the KLIC-score; an outcome predictor tool for prosthetic joint infections treated with debridement, antibiotics and implant retention. J Bone Jt Infect. (2018) 3:150-5. doi: 10.7150/jbji.21846

32. Jiménez-Garrido C, Gómez-Palomo JM, Rodríguez-Delourme I, DuránGarrido FJ, Nuño-Álvarez E, Montañez-Heredia E. The kidney, liver, index surgery and $\mathrm{C}$ reactive protein score is a predictor of treatment response in acute prosthetic joint infection. Int Orthop. (2018) 42:338. doi: 10.1007/s00264-017-3670-4

33. Luz CF, Vollmer M, Decruyenaere J, Nijsten MW, Glasner C, Sinha B. Machine learning in infection management using routine electronic health records: tools, techniques, and reporting of future technologies. Clin Microbiol Infect. (2020) 26:1291-9. doi: 10.1016/j.cmi.2020.02.003

34. Shohat N, Goswami K, Tan T, Yayac M, Soriano A, Sousa R, et al. Who will fail following irrigation and debridement for periprosthetic joint infection: a machine learning based validated tool. Bone Joint J. (2020) 11-9. doi: 10.1302/0301-620X.102B7.BJJ-2019-1628.R1

35. Gardner J, Gioe TJ, Tatman P. Can this prosthesis be saved? Clin Orthop Relat Res. (2011) 469:970-76. doi: 10.1007/s11999-010-1417-2

36. Kavolus JJ, Cunningham DJ, Eftekhary N, Ting NT, Griffin WL, Fehring TK. Fate of two-stage reimplantation after failed irrigation and debridement for periprosthetic knee infection. Clin Orthop Relat Res. (2011) 469:1825. doi: 10.1007/s11999-010-1434-1

37. Wouthuyzen-Bakker M, Sebillotte M, Lomas J, Kendrick B, Palomares FB, Murillo $\mathrm{O}$, et al. Timing of implant-removal in late acute periprosthetic joint infection: a multicenter observational study. J Infect. (2019) 79:199205. doi: 10.1016/j.jinf.2019.07.003

38. Ferry T, Leboucher G, Fevre C, Herry Y, Conrad A, Josse J, et al. Salvage debridement, antibiotics and implant retention ("DAIR") with local injection 
of a selected cocktail of bacteriophages: is it an option for an elderly patient with relapsing Staphylococcus aureus prosthetic-joint infection? Open Forum Infect Dis. (2018) 5:ofy269. doi: 10.1093/ofid/ofy269

39. El Haj C, Murillo O, Ribera A, Vivas M, Garcia-Somoza D, Tubau F, et al. Comparative efficacies of cloxacillin-daptomycin and the standard cloxacillin-rifampin therapies against an experimental foreignbody infection by methicillin-susceptible Staphylococcus aureus. Antimicrob Agents Chemother. (2014) 58:5576-80. doi: 10.1128/AAC.02 681-14

40. Utsui Y, Ohya S, Magaribuchi T, Tajima M, Yokota T. Antibacterial activity of cefmetazole alone and in combination with fosfomycin against methicillinand cephem-resistant Staphylococcus aureus. Antimicrob Agents Chemother. (1986) 30:917-22. doi: 10.1128/AAC.30.6.917

41. Miró JM, Entenza JM, Del Río A, Velasco M, Castañeda X, de la Mària CG, et al. High-dose daptomycin plus fosfomycin is safe and effective in treating methicillin-susceptible and methicillin-resistant Staphylococcus aureus endocarditis. Antimicrob Agents Chemother. (2012) 56:4511-5. doi: 10.1128/AAC.06449-11

42. Parra-Ruiz J, Bravo-Molina A, Pena-Monje A, Hernandez-Quero J. Activity of linezolid and high-dose daptomycin, alone or in combination, in an in vitro model of Staphylococcus aureus biofilm. J Antimicrob Chemothe. (2012) 67:2682-5. doi: 10.1093/jac/dks272

43. Garrigos C, Murillo O, Lora-Tamayo J, Verdaguer R, Tubau F, Cabellos $\mathrm{C}$, et al. Fosfomycin-daptomycin and other fosfomycin combinations as alternative therapies in experimental foreign-body infection by methicillinresistant Staphylococcus aureus. Antimicrob Agents Chemother. (2013) 57:60610. doi: 10.1128/AAC.01570-12

44. Mihailescu R, Tafin UF, Corvec S, Oliva A, Betrisey B, Borens O, et al. High activity of Fosfomycin and Rifampin against methicillinresistant staphylococcus aureus biofilm in vitro and in an experimental foreign-body infection model. Antimicrob Agents Chemother. (2014) 58:254753. doi: 10.1128/AAC.02420-12

45. Corvec S, Furustrand Tafin U, Betrisey B, Borens O, Trampuz A. Activities of fosfomycin, tigecycline, colistin, and gentamicin against extended-spectrum$\beta$-lactamase-producing Escherichia coli in a foreign-body infection model. Antimicrob Agents Chemother. (2013) 57:1421-7. doi: 10.1128/AAC.01718-12

46. Escudero-Sanchez R, Senneville E, Digumber M, Soriano A, Del Toro MD, Bahamonde A, et al. Suppressive antibiotic therapy in prosthetic joint infections: a multicentre cohort study. Clin Microbiol Infect. (2019) 26:499505. doi: 10.1016/j.cmi.2019.09.007

47. Pouderoux C, Becker A, Goutelle S, Lustig S, Triffault-Fillit C, Daoud F, et al. Subcutaneous suppressive antibiotic therapy for bone and joint infections: safety and outcome in a cohort of 10 patients. J Antimicrob Chemother. (2019) 74:2060-64. doi: 10.1093/jac/dkz104

48. Tornero E, Morata L, Martinez-Pastor JC, Angulo S, Combalia A, Bori G, et al. Importance of selection and duration of antibiotic régimen in prosthetic joint infections treated with debridement and implant retention. J Antimicrob Chemother. (2016) 71:1395-401. doi: 10.1093/jac/dkv481

Conflict of Interest: The authors declare that the research was conducted in the absence of any commercial or financial relationships that could be construed as a potential conflict of interest.

Copyright $\odot 2021$ Wouthuyzen-Bakker, Shohat, Parvizi and Soriano. This is an open-access article distributed under the terms of the Creative Commons Attribution License (CC BY). The use, distribution or reproduction in other forums is permitted, provided the original author(s) and the copyright owner(s) are credited and that the original publication in this journal is cited, in accordance with accepted academic practice. No use, distribution or reproduction is permitted which does not comply with these terms. 